\title{
Editorial: Nanofilms and Nanostructures for Energy Applications
}

\author{
Mariana Amorim Fraga ${ }^{1 *}$, Rodrigo Savio Pessoa ${ }^{2}$ and Ghulam Ali $^{3}$ \\ ${ }^{1}$ Department of Biomedical Engineering, Federal University of São Paulo (UNIFESP), São José dos Campos, Brazil, ${ }^{2}$ Plasmas and \\ Processes Laboratory, Aeronautics Institute of Technology (ITA), São José dos Campos, Brazil, ${ }^{3}$ USPCAS-E, National University \\ of Sciences and Technology (NUST), Islamabad, Pakistan
}

Keywords: nanofilms, nanostructures, energy conversion, energy harvesting, energy storage

Editorial on the Research Topic

Nanofilms and Nanostructures for Energy Applications

Since the beginning of the nanotechnology era, many efforts have been made to fully understand and explore the potential of nanoscale science and technology. In the last decades, we witness how advances in this multidisciplinary field are increasingly present in our daily lives. Among its areas of application, the use of nanomaterials to improve the performance of energy conversion, harvesting, and storage devices is one of the hot topics. The outstanding properties of different types of nanomaterials provide a wide opportunity for researchers and designers to develop more efficient energy technologies. Currently, nanomaterials are explored in several energy devices, such as solar cells, fuel cells, supercapacitors, batteries, among others. In this context, a significant progress in the synthesis, characterization and processing of nanomaterials for energy applications is being achieved. This research topic collected five articles, three original research articles, one methods article and one review article, which cover recent findings and developments in nanofilms and nanostructures for energy applications. Relevant themes in nanotechnology for energy research field were explored in these publications, namely electrodeposited $\mathrm{Ni}-\mathrm{Cu}$ foams as electrodes for supercapacitors, optical properties of plasmonic nanostructures, growth of titanium dioxide $\left(\mathrm{TiO}_{2}\right)$ thin films by atomic layer deposition (ALD), fabrication of high-efficiency hydrogenated amorphous-silicon (a-Si:H) solar cells and preparation of supported metal catalysts using non-thermal plasmas. Figure 1 illustrates these themes.

The first original research article reports the results of the electrochemical characterization of electrodeposited porous nickel-copper metallic foams on stainless steel substrate by the dynamic hydrogen bubbling template technique aiming at its use as an electrode for supercapacitors. The effect of working parameters, such as the applied current density and deposition time, on the surface morphology and properties of the electrodeposited layers were also discussed. It was observed that the $\mathrm{Ni}-\mathrm{Cu}$ foams have the potential to be used as an ideal electrode material for supercapacitors (Abdelfatah et al.).

The second original research article presents a theoretical investigation on the optical properties of metallic nanostructures and how they support plasmon resonances that can be tuned in the desired range of the solar spectrum by changing their size, shape, and surrounding environment. This study focused on two different systems: isolated and coupled gold metal nanospheres. The results indicated that the simulation of extinction spectrum of these nanostructures can be further extended to coupled nanospheroids, coupled nanorods, and chains of nanoparticles, which can be used in several applications such as solar stills, solar collectors, and solar cells (Pathak).

In the scope of thin film technology, the third original research article presents a detailed theoretical and experimental study on the impact of the precursor's pulse time on the growth per 


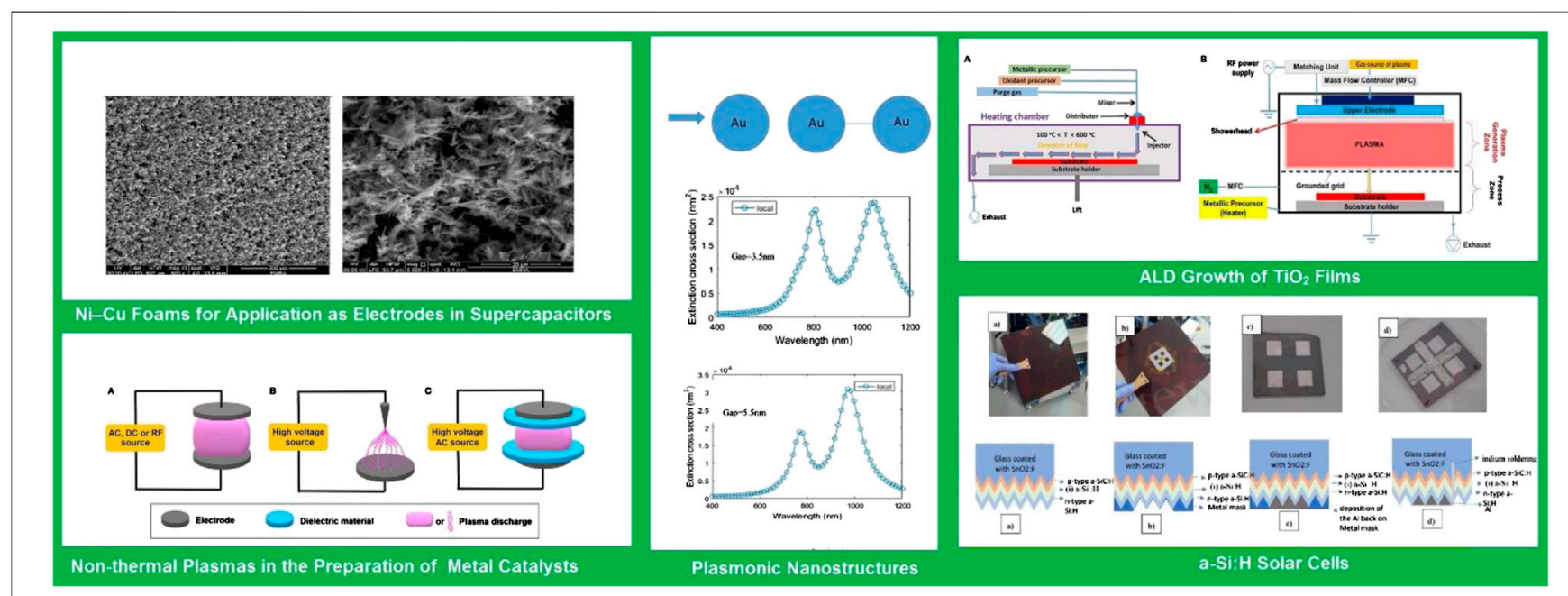

FIGURE 1 | Illustration of the themes addressed in the research topic nanofilms and nanostructures for energy applications.

cycle (GPC) and the crystallinity quality of atomic layer deposited $\mathrm{TiO}_{2}$ thin films on $\mathrm{Si}(100)$ and fluorine tin oxide (FTO) substrates (Chiappim et al.). ALD technique provides a unique tool to grow thin films with excellent conformity and thickness control down to atomic levels, which has motivated its use in solar cells research. Besides its application in well-established solar cell concepts such as c-Si and CIGS (copper indium gallium selenide), ALD technique has also been used to produce nanostructured thin films for emerging solar cell concepts such as dye-sensitized solar cells (DSSC) and perovskite-based solar cells. Among the most studied ALD films for such applications, the $\mathrm{TiO}_{2}$ has been highlighted.

The methods article proposes a promising shadow masking technique to fabricate high-efficiency a-Si:H using plasma enhanced chemical vapor deposition (PECVD). The solar cell fabrication process based on this technique was described in detail. The influence of the different masking methods on the cell parameters was also discussed. The results show that the use of proposed shadow masking technique using $\mathrm{p}-\mathrm{i}-\mathrm{n}$ structure deposited on large-area substrates, followed by the deposition of back contact through a metal mask, and by ultrasonic welding of indium is a good alternative to laser scribing in the laboratory scale (Alaoui et al.).

The review article discusses on the use of non-thermal plasmas in the preparation or pretreatment of supported metal catalysts for fuel conversion in automotive systems. Several studies show that the use of non-plasma technology can be an advantageous process in supported metal catalysts due to the dispersion of metallic nanoparticles present in the catalyst surface, causing stronger interaction between active phase and support. Thus, higher activity, selectivity, and stability, as well as lower activation temperature, are observed. This review provides the reader with an overview showing the synergistic effects between plasma environment and heterogeneous catalysis, the methods of preparation of supported metal catalysts, detailing several plasma-assisted processes, and presenting the main results of plasma-prepared supported metal catalysts for fuel conversion (dos Santos et al.).

Overall, the articles published in this research topic report and discuss various important and current issues related to nanomaterials for energy applications. Different materials, techniques and applications were addressed making this collection of articles a good reference to researchers working on these subjects.

\section{AUTHOR CONTRIBUTIONS}

All authors listed have made a substantial, direct, and intellectual contribution to the work and approved it for publication.

Conflict of Interest: The authors declare that the research was conducted in the absence of any commercial or financial relationships that could be construed as a potential conflict of interest.

Copyright (c) 2021 Fraga, Pessoa and Ali. This is an open-access article distributed under the terms of the Creative Commons Attribution License (CC BY). The use, distribution or reproduction in other forums is permitted, provided the original author $(s)$ and the copyright owner $(s)$ are credited and that the original publication in this journal is cited, in accordance with accepted academic practice. No use, distribution or reproduction is permitted which does not comply with these terms. 